livraisons

d'Histoire

de l'Architecture

\section{Livraisons de l'histoire de l'architecture}

24 | 2012

Le phare et l'architecte

\title{
Yves Hémar, Henry Auffret: variations autour du phare breton
}

Yves Hémar and Henry Auffret: Variations on the Breton Lighthouse

Yves Hémar, Henry Auffret: Verschiedene Betrachtungen über den bretonischen Leuchtturmtyp

Hervé Raulet

\section{(2) OpenEdition}

Journals

Édition électronique

URL : http://journals.openedition.org//ha/99

DOl : $10.4000 /$ /ha.99

ISSN : 1960-5994

Éditeur

Association Livraisons d'histoire de l'architecture - LHA

Édition imprimée

Date de publication : 19 décembre 2012

Pagination : 125-148

ISSN : 1627-4970

\section{Référence électronique}

Hervé Raulet, "Yves Hémar, Henry Auffret: variations autour du phare breton », Livraisons de l'histoire de l'architecture [En ligne], 24 | 2012, mis en ligne le 16 avril 2015, consulté le 01 mai 2019. URL : http:// journals.openedition.org/lha/99 ; DOI : 10.4000/lha.99

Ce document a été généré automatiquement le 1 mai 2019.

Tous droits réservés à l'Association LHA 


\title{
Yves Hémar, Henry Auffret: variations autour du phare breton
}

\author{
Yves Hémar and Henry Auffret: Variations on the Breton Lighthouse \\ Yves Hémar, Henry Auffret: Verschiedene Betrachtungen über den bretonischen \\ Leuchtturmtyp
}

Hervé Raulet

1 En Bretagne, la construction des phares après de la seconde guerre mondiale concerne quasi exclusivement le littoral Nord, de Saint-Malo à Perros-Guirec, cette partie de la côte ouverte sur la Manche et relativement proche de la Normandie ayant été, plus que d'autres, touchée par les destructions. Les principaux phares, qui avaient été minés à partir de 1942 par les troupes allemandes, furent systématiquement dynamités par cellesci au moment de l'offensive alliée d'août 1944: le Grand-Jardin, Rochebonne et la Balue à Saint-Malo, le cap Fréhel à Plévenon, Bodic et la Croix près de Lézardrieux, les Héaux de Bréhat au large de Pleubian, le Paon et le Rosédo sur l'île de Bréhat, Ploumanac'h, les Sept-Iles, les Roches-Douvres à quarante kilomètres des côtes, sans compter bien d'autres feux de moindre importance.

2 La reconstruction de ces phares s'engage très rapidement, la plupart des études préliminaires débutant fin 1944. Le service des Phares et balises établit généralement un feu provisoire à proximité immédiate de l'ouvrage détruit, afin de disposer du temps nécessaire à la mise en œuvre du nouveau phare, l'allumage de celui-ci intervenant, selon les sites, entre 1947 et 1954. Deux architectes contribuent étroitement à l'entreprise: Yves Hémar, qui se consacre aux phares de la Balue et de Rochebonne mais surtout à celui du cap Fréhel, et Henry Auffret qui, seul ou associé à un troisième architecte, Joël Hardion, signe la quasi-totalité des autres ouvrages, en particulier le Grand-Jardin et les RochesDouvres ${ }^{1}$. 


\section{La reconstruction de Saint-Malo}

Yves Hémar (1886-1955) et Henry Auffret (1919-1997) sont, à des degrés divers, des architectes reconnus au plan régional qui, à l'époque, participent à l'un des grands chantiers français de l'après-guerre, celui de la reconstruction de la cité historique de Saint-Malo détruite aux trois quarts lors de l'offensive alliée d'août 1944. Tous deux Malouins et anciens élèves de l'École nationale des Beaux-arts de Paris, ils se succèdent au poste d'architecte municipal de Saint-Malo: Hémar incarne toute la période de l'entredeux-guerres alors qu'Auffret assume cette charge à partir de 1945, ses fonctions l'amenant à construire de nombreux bâtiments publics et élaborer plusieurs programmes d'envergure dans sa ville natale durant une vingtaine d'années. La participation d'Yves Hémar à la reconstruction de Saint-Malo apparaît moins déterminante, surtout si l'on considère la notoriété de cet architecte avant-guerre ${ }^{2}$. Son rôle se limite principalement à la réalisation, comme architecte d'opération, à trois îlots d'habitations dans l'intra muros ${ }^{3}$ (ill. 1).

\section{1: Saint-Malo, immeuble d'habitation}

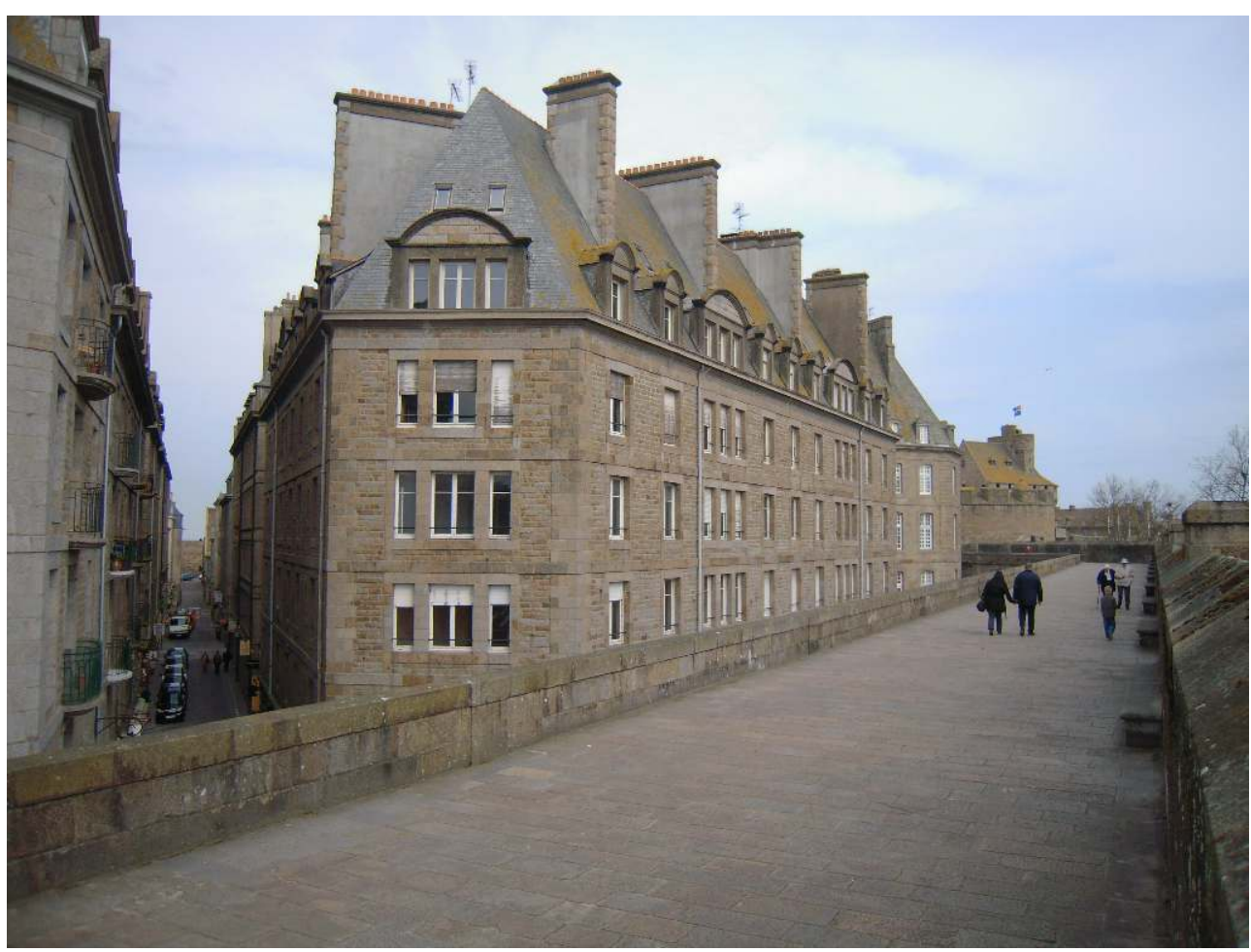

Rue Jacques Cartier (îlot 58 du plan de reconstruction, Hémar, architecte, ca 1950), vu depuis les remparts

Cl. Hervé Raulet

4 Ces immeubles s'inscrivent dans le programme d'ensemble mis en œuvre entre la fin 1944 et 1960 sous la direction des architectes Marc Brillaud de Laujardière ${ }^{4}$ puis Louis Arretche, programme guidé par la volonté de «reconstruire à l'identique » la cité historique, ce principe ne signifiant pas une reconstitution exacte, au sens archéologique, de la ville disparue - seul un nombre très limité de façades correspondant à des monuments historiques en ont bénéficié - mais un respect de la forme ancienne de celle- 
ci à l'intérieur de ses remparts préservés de la destruction: les gabarits des nouveaux îlots une fois définis, naît une trame générale respectueuse du site et de son patrimoine ancien façonné par les grands hôtels de cinq ou six niveaux d'élévation érigés sous le règne de Louis XIV, à l'intérieur de laquelle chaque opérateur s'insère en proposant pour son immeuble une modulation stylistique, l'ensemble définissant une sorte de "style malouin " historicisant. Ainsi, tout en faisant sien le modèle classique de référence (appareils de granite, ordonnancement régulier des travées, toits pentus dominés par de hautes souches de cheminées), Hémar rappelle, par touches discrètes, son attachement à certaines de ses formules d'inspiration régionaliste abondamment utilisées dans l'entredeux-guerres: lucarnes à frontons plein cintre, oculi, encadrements des baies et chaînes d'angles à bossages, etc. En dehors de la ville ancienne, l'architecte participe à quelques réalisations dans le domaine du logement (lotissement bon marché de Riancourt à SaintServan, villa Lublanjack avenue Pasteur dans laquelle il renoue avec les motifs décoratifs celtiques comme le triskel) et, dans l'architecture publique cette fois, dessine quelques équipements tels la nouvelle gare maritime inaugurée en $1951^{5}$.

5 L'œuvre d'Hémar la plus marquante de l'après-guerre, le phare du cap Fréhel, reste toutefois géographiquement extérieure à Saint-Malo, même si le site de Fréhel est historiquement lié à l'ancienne cité corsaire depuis le milieu du XVII e siècle. Ce sont en effet les armateurs et commerçants malouins qui financèrent la construction et l'entretien des premiers feux sur le cap, afin de signaler aux bateaux venant de l'ouest qu'ils approchaient de leur but. Le deuxième phare du cap Fréhel, qui fonctionna de 1702 à $1847^{6}$ et qui se dresse aujourd'hui encore aux côtés du phare d'Yves Hémar ${ }^{7}$, fut construit par Jean-Siméon Garangeau (1647-1741), nommé par Vauban ingénieur en chef et directeur des fortifications de Saint-Malo en 1691, son fonctionnement restant pris en charge par les malouins jusqu'en 1792, année où il fut transféré à l'État (ministère de la Marine) comme tous les autres phares, amers, bouées et balises du littoral français.

\section{Yves Hémar, un « apôtre du régionalisme »}

6 Hémar fut pendant plus de vingt ans l'un des chefs de file de l'architecture régionaliste en Bretagne, bien que son œuvre se concentrât sur un secteur géographiquement limité au littoral de la côte d'Émeraude, de Fréhel à Cancale. Avec le phare de Fréhel, il renoue sans doute avec une certaine liberté d'action et des conditions matérielles suffisamment "généreuses" et stimulantes, assez comparables à celles qui présidèrent à ses commandes d'avant-guerre lorsqu'il était un architecte introduit auprès des institutions et de la riche clientèle privée fréquentant la côte d'Émeraude. Il se retrouve en outre à quelques encablures de son premier grand chantier, la station balnéaire de Sables-d'Orles-Pins créée à partir de 1923 par le promoteur malouin Roland Brouard. Hémar, alors âgé de trente-sept ans, est connu comme architecte municipal de Saint-Malo et dirige un cabinet privé ouvert trois ans plus tôt dans cette même ville. Il a réalisé plusieurs villas sur la côte pour une clientèle fortunée et sa réputation d'architecte " fort distingué» et " d'homme d'esprit, d'érudition et de talent $»^{8}$ est déjà, semble-t-il, bien établie 9 . Brouard lui confie la réalisation de la plupart des hôtels et commerces situés au cœur de la station naissante, comptant sur sa notoriété et son entregent pour attirer de nouveaux clients désireux de se faire construire une résidence de "bon goût ", c'est-à-dire conforme au "style régional " qu'est sensé incarner l'architecte ${ }^{10}$, un peu à la manière du brestois Gaston Chabal (1882-1965) œuvrant quelques années plus tôt à Morgat (Finistère). Car 
l'architecture «bretonne » de cette époque s'apparente encore majoritairement au style balnéaire d'influence anglo-normande alors en vogue, avec sa part de pittoresque et d'éclectisme. La Bretagne n'avait pas connu jusqu'ici de style véritablement néo-régional et celui-ci était précisément en train de se construire au milieu de ces années $1920^{11}$. Les immeubles collectifs conçus par Hémar à Sables-d'Or présentent une grande variété, depuis le lisse et immaculé hôtel des Arcades construit en 1924 et exempt de toute référence "régionale" - si ce n'est peut-être dans une acception coloniale puisque ce bâtiment de plan hexagonal aux élévations rythmées d'arcades n'est pas sans rappeler l'architecture administrative du Maroc des années $1910^{12}$ - jusqu'aux hôtels et commerces à faux colombages inspirés des stations normandes (hôtel des Ajoncs d'or, hôtel des Dunes, les Portiques, etc.).

7 Ses villas (Castel Breiz, Lantzenberg, Les Pins...) sont porteuses d'une grammaire plus spécifiquement «bretonne », telle qu'elle s'affirme dans la province à la suite du succès du pavillon breton Ty Breiz à l'Exposition des arts décoratifs et industriels modernes de Paris en $1925^{13}$, auquel Hémar prend une part active ${ }^{14}$. Il avait découvert durant ses études les théories architecturales et décoratives élaborées par l'Art \& Crafts Exhibition Society ainsi que leur mise en œuvre par les architectes Philip Webb dans la seconde moitié du $\mathrm{XIX}^{\mathrm{e}}$ siècle (The Red House) ou Charles Rennie Mackintosh à Glasgow vers 1900 (Windyhill Cottage), qui s'inspiraient des modèles de la maison rurale anglaise ou du manoir écossais. Il puise quant à lui dans le répertoire formel des manoirs bretons des $\mathrm{XVI}^{\mathrm{e}}$ et $\mathrm{XVII}^{\mathrm{e}}$ siècles ${ }^{15}$ tout en incorporant des éléments du style balnéaire, associant avec subtilité granite et grès d'extraction locale, faux pan-de-bois en ciment peint, hautes toitures débordantes surmontant des volumes trapus et bien individualisés, baies de formes variées, souches de cheminées puissantes, lucarnes à frontons cintrés, tours demi hors-œuvre, porches d'entrée, chevronnières à crossettes, etc. Ce régionalisme syncrétique, apparu dès les premières maisons de villégiature édifiées par l'architecte ${ }^{16}$, s'affirme désormais de manière plus ostensible dans ses villas des années 1925-1930. Un autre exemple éloquent nous est donné par la villa Le Revenant, construite en 1928 à Saint-Lunaire pour la baronne de Gargan, où l'architecte s'empare de l'archétype du manoir breton (matériaux, composition générale, décor) pour en donner une relecture toute moderne, la multiplication des baies largement ouvertes sur la mer traduisant la volonté de satisfaire les goûts de la commanditaire pour le confort et un art de vivre qui prenne en compte la relation avec le paysage (ill. 2). 


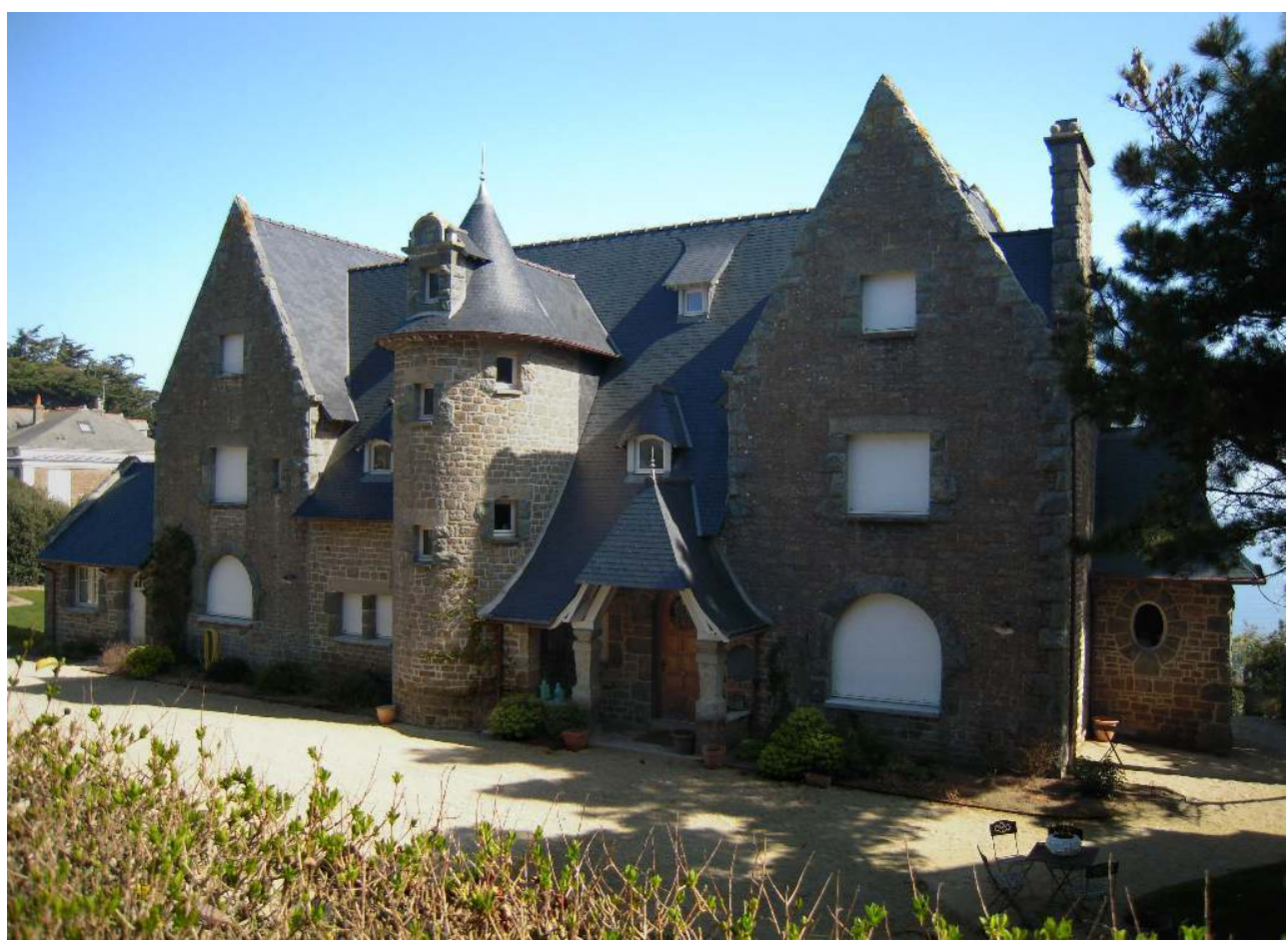

Hémar, architecte, 1928

Cl. Hervé Raulet

8 Cette rusticité savante et épurée du « style Hémar » rencontre un vif succès auprès de la clientèle aisée de la côte d'Émeraude, séduite à la fois par son originalité et son attachement rassurant aux valeurs traditionnelles, alors que des débats souvent vifs opposent les tenants du régionalisme aux promoteurs du modernisme: tandis que ces derniers cherchent à s'écarter des anciennes formules et défendent une conception universelle de l'architecture, débarrassée de tout déterminisme local et de toute ornementation au profit d'un rationalisme constructif où prime la fonction, les premiers considèrent que la création moderne doit puiser aux sources de l'histoire d'un pays, d'une région, et que les caractéristiques naturelles du lieu (situation géographique, relief, climat, matériaux, etc.) expliquent et justifient le type d'architecture qui y est produite, accordant donc à l'apparence une place essentielle. Journaux locaux et revues spécialisées des années 1920-1930 vantent ainsi les mérites des productions de l'architecte malouin:

«Monsieur Yves Hémar, archéologue distingué, collectionneur hors pair, créateur du plus intéressant des Musées bretons, représente l'esprit régionaliste. Il excelle à adapter à des programmes modernes les plus ingénieux pastiches des architectures du passé breton et normand. Granit et pan-de-bois, pignons aigus et mouvementés recouverts d'ardoises, souches de cheminée monumentales concourent à la variété des silhouettes, croisées en arc surbaissé à petits bois nombreux, dont la couleur blanche se détache sans heurt sur le fond gris de la pierre, tels sont les moyens dont Monsieur Yves Hémar use avec une incontestable maitrise. $»^{17}$

«Les maisons que Monsieur Yves Hémar a campées en avant-garde de nos plages face aux marées ont cette physionomie délivrée que le sommeil donne aux hommes. Elles sont bonnement de vieux manoirs bourrus qui, leurs muscles de granit bien bandés, luttent contre le vent. Leur façade est oblique en effet, comme pour assurer l'assiette de l'édifice, l'appareil est rustaud, la pierre brute. Elles ont une figure qu'on est habitué à voir dès le premier coup d'œil dans le paysage breton. $»^{18}$ 
«La villa Terouanne ${ }^{19}$ est de M. Yves Hémar dont la Construction moderne a déjà publié diverses productions. Tous les sains principes des constructions du MoyenÂge se retrouvent ici alliés au raffinement moderne. Quel parti remarquable les architectes de cette région ont su tirer de leurs matériaux locaux! [...] Élégance dans le solide, c'est ainsi que nous pouvons continuer à les caractériser, souci d'une construction robuste dans un pays ou les villas sont abandonnées plusieurs mois, recherche du détail pittoresque et aussi du confort auquel les propriétaires sont habitués, ce sont là des qualités qu'on ne saurait méconnaître. $»^{20}$

9

Ou encore:

"Yves Hémar n'est pas seulement un très moderne architecte dont les procédés dépassent souvent en heureuse audace les méthodes américaines, c'est un apôtre $\mathrm{du}$ régionalisme et la France compte peu de défenseurs aussi ardents de ses richesses provinciales. $»^{21}$

10

réalise également, durant les années trente, trois immeubles de logements sur la digue du Sillon à Saint-Malo (Ker Armel, L'Émeraude, La Frégate) où, privé en quelque sorte du cadre référentiel que constitue la demeure manoriale, et probablement contraint par l'économie des projets, il imprime sa marque avec plus de discrétion, s'en tenant à quelques motifs essentiellement décoratifs (faux pan-de-bois en partie haute de Ker Armel, encadrement des portes d'entrée, traitement d'un escalier extérieur...). Ces immeubles, en particulier L'Émeraude et La Frégate, constituent, par leur ordonnancement régulier et leur relatif dépouillement, un contrepoint quasi moderniste et assez inhabituel dans la production de l'architecte. Ils démontrent peut-être a contrario combien la production la plus spectaculaire et la plus connue d'Hémar à cette époque, c'est-à-dire ses villas, est conditionnée par la demande d'une clientèle revendiquant une forme d'assimilation à la "griffe » d'un architecte en vue, entretenant ainsi, dans une démarche somme toute assez conservatrice, la perpétuation d'un style.

\section{L'architecture publique d'Yves Hémar}

La production de cet " apôtre du régionalisme » ne se limite pas à l'architecture domestique mais concerne également les bâtiments publics. Les promoteurs du régionalisme dans l'entre-deux-guerres attachent une grande importance à cette catégorie d'édifices, considérant qu'ils sont, de par leur fonction représentative, les dépositaires privilégiés de traditions constructives et des vecteurs emblématiques de leurs conceptions sur l'art et l'architecture modernes. L'héritage architectural de la Bretagne est, dans ce domaine, essentiellement religieux, avec une prédominance du gothique dont le rayonnement perdure jusque tard dans le XVI ${ }^{\mathrm{e}}$ siècle ${ }^{22}$. Dans ces années 1920 et 1930, plusieurs architectes bretons construisent des églises et des chapelles où les problématiques modernistes et régionalistes se nourrissent mutuellement pour renouveler, avec talent, l'architecture sacrée de la région: on peut citer Georges-Robert Lefort (1875-1954) avec le grand séminaire de Saint-Brieuc (1925), James Bouillé (1894-1945), auteur de plusieurs édifices remarquables dont l'église Sainte-Thérèse à Saint-Brieuc (1930) et la chapelle de l'institution Saint-Joseph à Lannion $(1936)^{23}$ ou encore, dans un registre plus modeste, Charles Coüasnon avec sa petite chapelle de l'Hermitage à Goven (1936).

Mis à part la reconstruction du chœur et du transept de l'église de Rocabey à Saint-Malo, intervention peu significative au demeurant, Hémar ne s'intéresse pas à l'architecture religieuse. Il est par contre l'auteur de plusieurs bâtiments civils, à Saint-Malo bien sûr mais aussi dans plusieurs villes de la région: bureaux de poste, établissements pour 
enfants, équipements culturels et de loisirs, etc. Dans le domaine civil, l'évocation de modèles anciens proprement régionaux est d'emblée moins évidente que dans l'architecture domestique ou religieuse, les contraintes de programmes se voulant d'abord fonctionnels pouvant favoriser un régionalisme « de façade » restreint au décor et au choix de matériaux locaux. Les bâtiments publics d'Hémar proposent quant à eux une synthèse plus complète et subtile entre utilitarisme, formes inspirées de l'ancien et ornementation, à l'image de l'hôtel des postes de Saint-Malo intra muros (1928) (ill. 3).

\section{3: Saint-Malo, hôtel des postes}

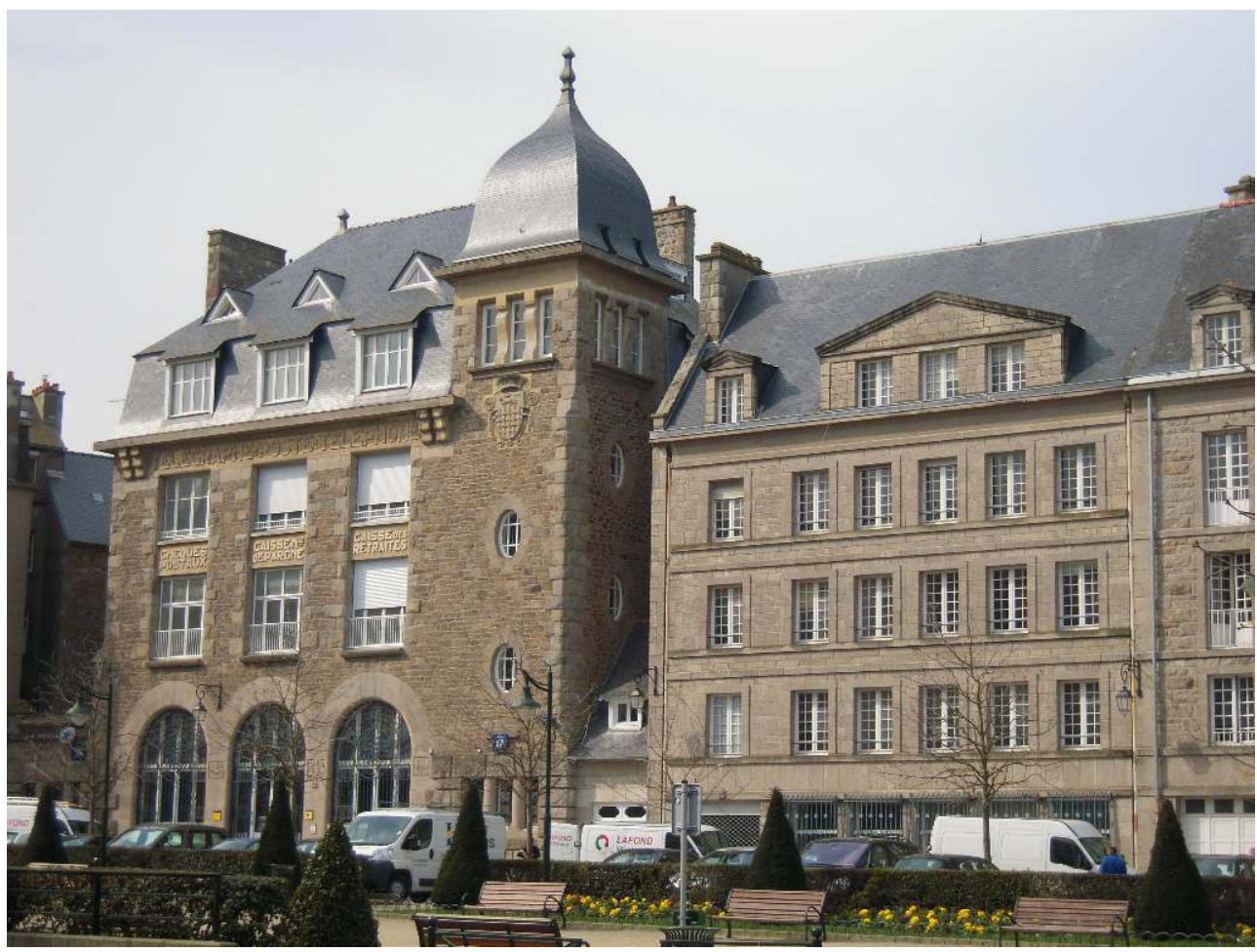

Hémar, architecte, 1928

Cl. Hervé Raulet

Avec sa haute façade en granite et ciment percée d'un triplet de baies en plein cintre et de larges fenêtres carrées, et flanquée d'une tour d'escalier percée d'oculi et dominée par un toit à l'impériale, le bâtiment extériorise une modernité toute fonctionnelle en affichant une double référence à la Bretagne du XVII e siècle, celle des hôtels urbains dont SaintMalo était richement dotée avant les destructions de la seconde guerre mondiale, et celle des grands manoirs ruraux. La franchise de son architecture est soulignée par un décor mêlant Art déco et motifs inspirés des broderies bretonnes, l'intérieur faisant la part belle aux mosaïques de l'atelier rennais d'Isidore Odorico. ${ }^{24}$ Hémar réalise deux ans plus tard le groupe scolaire du quartier malouin de Courtoisville, où le caractère fonctionnel de l'équipement est traduit plus nettement encore par une hiérarchisation claire des volumes, la répartition symétrique des bâtiments de part et d'autre d'un bloc central qui abrite l'accueil et le logement du directeur exprimant en outre une certaine solennité. Les emprunts à l'art breton (frontons et lucarnes cintrés, décor de spirales, etc.) accompagnent avec sobriété une composition générale établissant un lien étroit entre architecture et espace. 
Cette préoccupation de l'architecte d'adapter ses projets aux contraintes des programmes tout en s'adaptant au contexte local et régional, se retrouve dans deux équipements de la station balnéaire de Dinard implantés en front de mer, dans un environnement mêlant villas et activités maritimes: le club nautique (1932) et le muséum national d'histoire naturelle (1935). Le premier réinvestit le répertoire formel de la villa balnéaire régionaliste auquel l'architecte nous a habitués (corps de bâtiments différenciés, pignon en façade, bow-window, tourelle, arcades en plein cintre, etc.) alors que le second, qui procède de la transformation d'une ancienne villa à flanc de rocher ${ }^{25}$ pour y installer des laboratoires et deux aquariums ouverts au public, s'inscrit dans une veine beaucoup plus moderniste, de style "paquebot »: juxtaposition de volumes géométriques simples (cylindres, parallélépipèdes) reliés par des cheminements formant coursives, lignes courbes, fenêtres rondes ou carrées disposées en bandeaux, corniches saillantes, toitsterrasses, etc (ill. 4).

\section{4: Dinard, muséum national d'histoire naturelle}

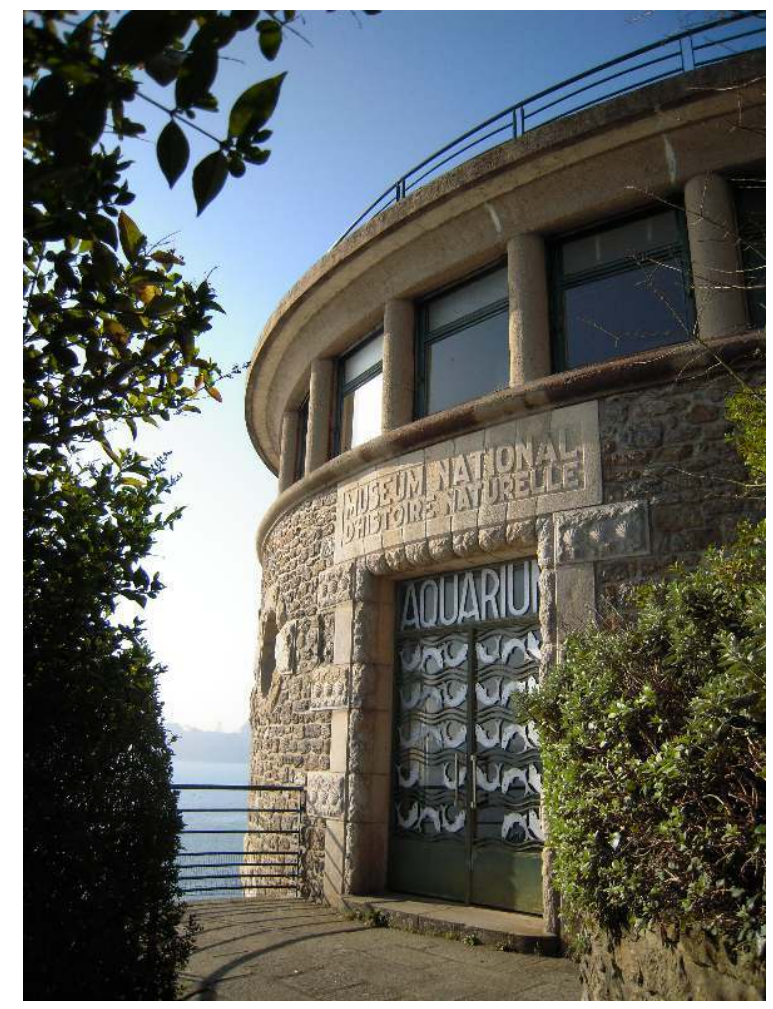

Hémar, architecte, 1935, entrée de l'aquarium

Cl. Hervé Raulet

15 Hémar emploie comme toujours le moellon équarri et la pierre de taille de granite associés au béton, la pierre rustiquée venant souligner les lignes de force des bâtiments (chaînes d'angles, baies, chevronnières, etc.). 


\section{Le phare du cap Fréhel, dernier grand chantier d'Yves Hémar}

Les équipements publics construits par Hémar après 1945 sont peu nombreux, tout d'abord en raison du fait qu'il n'est plus architecte de la ville de Saint-Malo après la guerre, ensuite parce que les autres commanditaires institutionnels ont sans doute préféré se tourner vers des maîtres d'œuvre jugés plus en phase avec les préoccupations du moment en matière d'architecture et d'urbanisme, qu'un architecte symbolisant, tant par son style et sa manière de travailler que par son influence passée, un entre-deuxguerres un peu daté. Mais c'est précisément ce style et cette réputation qui semblent avoir convaincu le Service des phares de s'assurer la contribution d'Hémar pour le cap Fréhel, outre le fait que l'architecte s'occupe également de la reconstruction à Saint-Malo des feux de la Balue (1948) et de Rochebonne (1948-1951) ainsi que de ses propres bâtiments administratifs également détruits par les bombardements. La reconstruction des phares du Nord-Bretagne s'inscrit en effet davantage dans un esprit de continuité que de rupture, traduisant une forme de conservatisme, non pas dans le domaine technique où l'évolution est la règle, mais dans l'architecture, l'immuabilité du modèle de la tour se renforçant de la volonté de l'administration de ne pas bouleverser l'image des phares auprès de la population, de même que le rapport entre ouvrages et sites d'implantation. Dans une note adressée le 19 juillet 1945 par le directeur du service des Phares et balises, André Gervais de Rouville, à son ingénieur en chef $\mathrm{H}$. Condemine, on perçoit toute la valeur accordée à l'esthétique du futur ouvrage qui doit pouvoir se comparer à celle des plus illustres témoins du patrimoine régional:

« $\mathrm{Au}$ moment de commencer la reconstruction de l'établissement de Fréhel, J'appelle votre attention sur son importance non seulement pour la grande navigation, mais aussi pour les touristes et pour le bon renom de notre pays, en raison de la richesse exceptionnelle de la côte d'Émeraude en sites appréciés des étrangers et en monuments historiques de haute valeur $»^{26}$.

17 Le rapport établi un an plus tard par l'ingénieur pour présenter le projet définitif, décrit ainsi l'aspect général de la tour:

"L'allure générale du phare est majestueuse et convient bien au caractère du site. Ses lignes sont classiques et s'apparentent à l'architecture militaire du XIV siècle. Certains éléments de détail permettent cependant d'éviter une monotonie trop austère. Le choix des matériaux de parement est primordial. Dans cette région bretonne, l'utilisation du granit s'impose. Ce matériau accentue le caractère robuste du bâtiment et s'harmonise parfaitement avec les rochers de la pointe [...]. Il sera taillé en très gros bossages. Cela donnera au bâtiment une allure puissante et favorisera des jeux de lumière d'un bel effet $"^{27}$.

Ce phare de premier ordre, allumé le $1^{\mathrm{er}}$ juillet 1950, adopte une composition symétrique dominée par une solide tour carrée de trente mètres couronnée de créneaux, centrée à la façade d'un bâtiment de plan allongé encadré par deux ailes en retour d'équerre et précédé d'une cour rectangulaire. La maçonnerie apparente de granite roux associe un appareil régulier de moellons et de pierres de taille rustiquées, ponctué de blocs de granite gris taillés en bossage. La partie inférieure du bâtiment est l'objet d'un traitement particulier qui la différencie de la volumétrie anguleuse de l'ensemble: la porte d'entrée en plein cintre, surmontée d'un oculus, est accostée d'un triplet d'arcades soutenues par des colonnes doubles en granite gris, selon une disposition inspirée de la serlienne (ill. 5). 


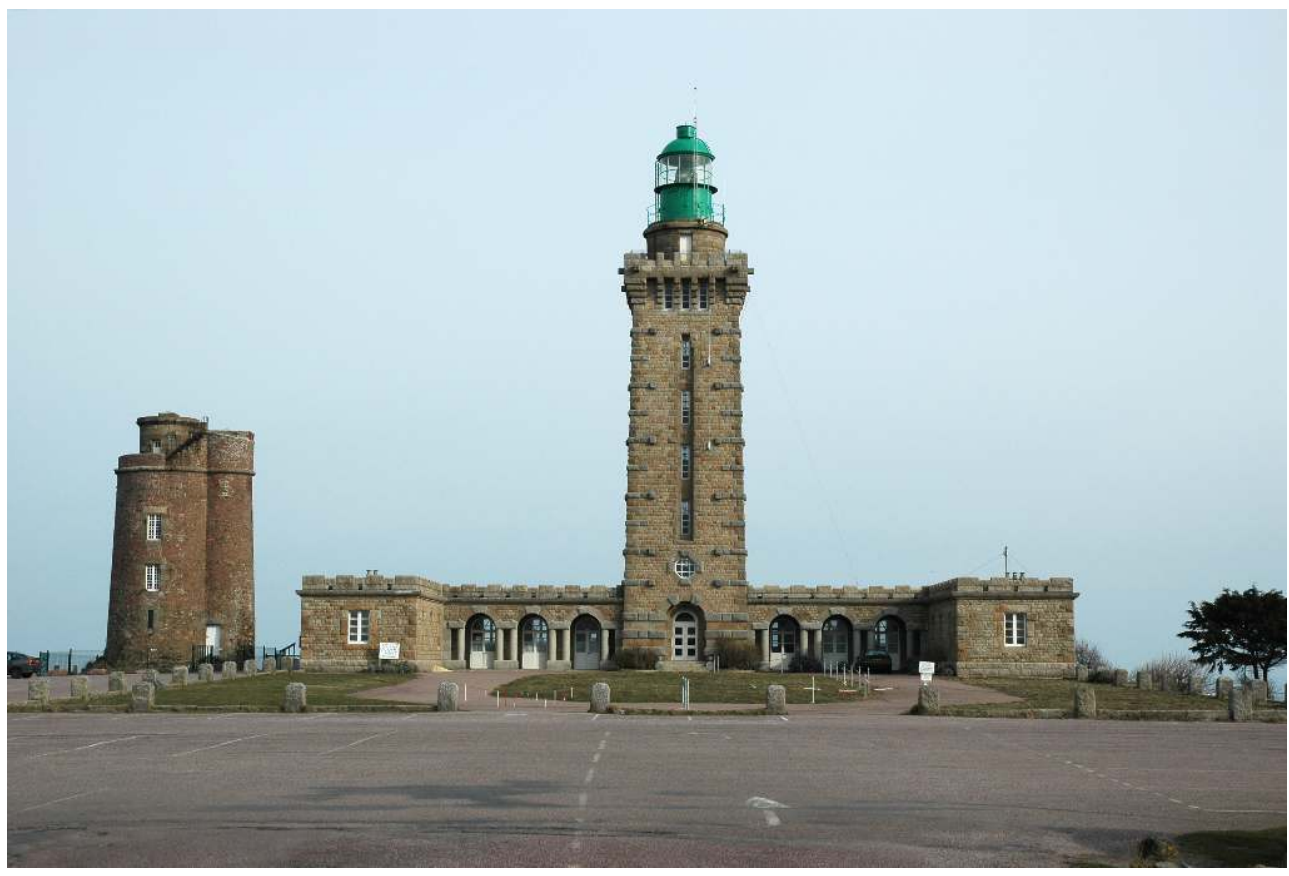

Hémar, architecte, 1950 et ancien phare ; Garangeau, ingénieur ; en fonction de 1702 à 1847 puis de 1944 à 1950

Cl. Hervé Raulet

Cet édifice élégant et soigné, au caractère à la fois rustique et raffiné propre à l'architecte, s'inscrit dans la continuité du phénomène de castellisation architecturale apparu au XIX ${ }^{\mathrm{e}}$ siècle avec le développement du balnéaire, Hémar concevant ici un pastiche italianisant de ces phares à tour carrée érigés aux XVIII ${ }^{\mathrm{e}}$ et XIX ${ }^{\mathrm{e}}$ siècles le long des côtes françaises ${ }^{28}$. Signalons également l'étroite parenté de style entre la tour du cap Fréhel et le nouveau phare de Ploumanac'h, petit ouvrage en granite rose dont les plans sont établis à la même période par l'ingénieur des Phares et balises Grattesat (de novembre 1945 pour les premières esquisses à octobre 1946 pour le projet définitif). Savoir lequel est antérieur à l'autre n'a que peu d'importance mais permet de souligner le succès d'un modèle qui se décline en multiples avatars jusqu'à une date récente. Le phare de Fréhel reste, en dépit de son style qui n'a rien de "breton ", une œuvre fidèle à l'esprit régionaliste, l'architecte opérant un retour sur l'histoire de cette typologie d'ouvrages pour en proposer une ultime variation, à la fois parfaitement moderne sur le plan de ses dispositions fonctionnelles et techniques (phare électrique, ascenseur, etc.) et traditionnelle du point de vue de son architecture. La démarche historiciste à laquelle Hémar nous a habitués trouve, avec ce phare, un terrain d'expression privilégié. Ouvrage quelque peu grandiloquent et anachronique en ce milieu de $\mathrm{XX}^{\mathrm{e}}$ siècle, il répond à l'attachement manifeste du commanditaire institutionnel pour ce XIXe siècle qui a vu se développer un grand réseau de signalisation moderne, constitué d'ouvrages fonctionnels, élégants et durables. Chant du cygne d'un créateur né lui-même au XIX ${ }^{e}$ siècle et travaillé par la question de l'héritage culturel, le phare du cap Fréhel s'érige ainsi comme un hommage appuyé à ces grands phares continentaux du siècle précédent et un testament architectural empreint de nostalgie d'un régionalisme finissant. 


\section{Un jeune architecte prolifique: Henry Auffret}

La démarche d'Henry Auffret, en ces lendemains de guerre, s'annonce bien différente, celle d'un jeune architecte s'affrontant très vite aux problématiques et défis de la reconstruction. Après quelques années à Paris, il devient dès octobre 1944 l'un des principaux collaborateurs de Marc Brillaud de Laujardière à Saint-Malo, avant d'être nommé architecte de cette ville l'année suivante, y exerçant dès lors un rôle majeur. Il construit à Saint-Malo de très nombreux équipements publics, en premier lieu l'hôtel des finances (1948) ${ }^{29}$ (ill. 6) et le groupe scolaire du Grand-Bé (1951) (ill. 7) situés tous deux au cœur de la cité historique, proposant pour ces immeubles une architecture rigoureuse où la volumétrie étirée et le rythme des façades, scandé par l'intervalle des éléments porteurs et des baies disposées en bandeaux, se distingue nettement du parti de reconstitution adopté pour les immeubles de logement, tout en s'intégrant à lui par le choix des mêmes matériaux, granite, ciment moulé imitant la pierre, béton.

\section{6: Saint-Malo, hôtel des finances}

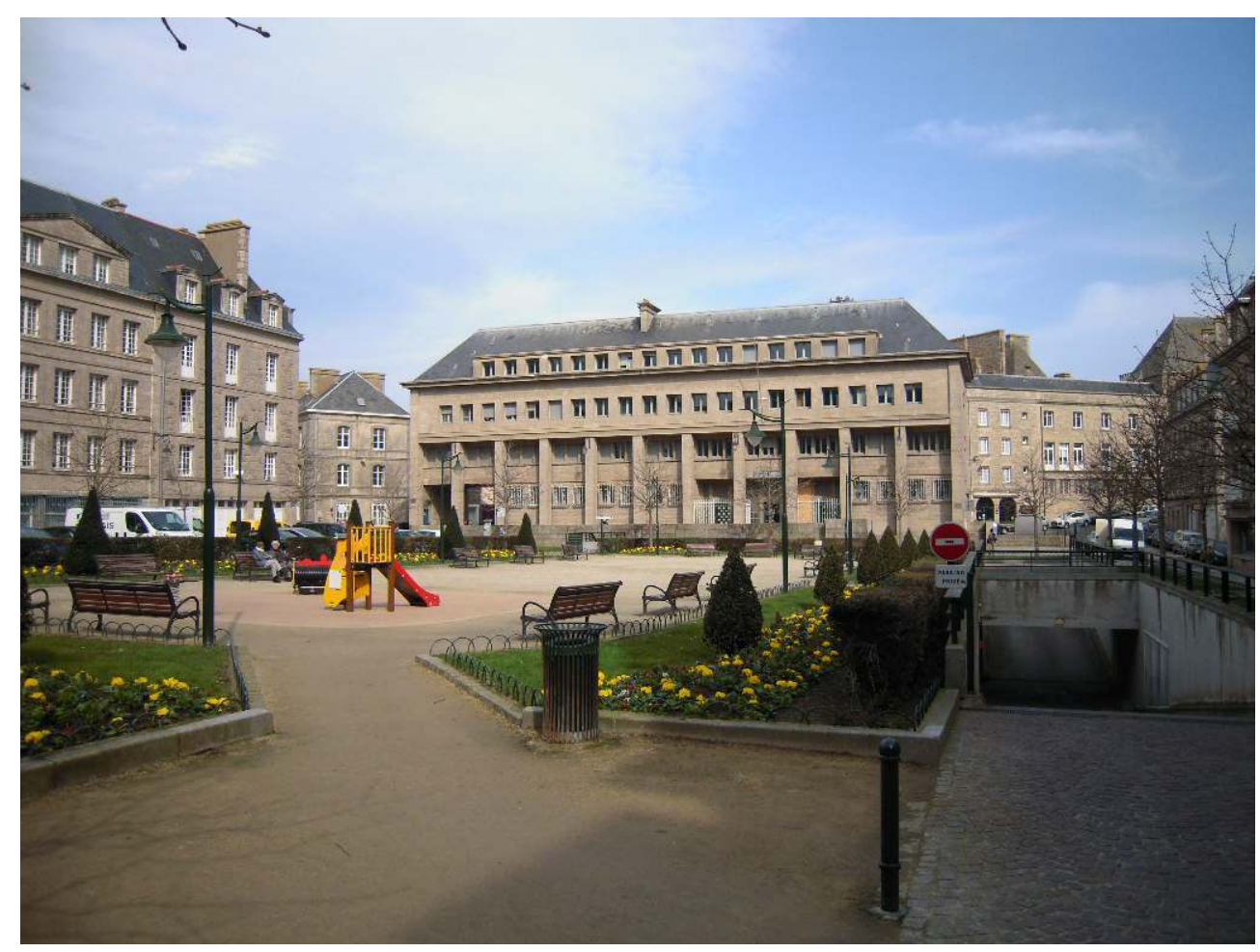

Auffret, architecte, 1948

Cl. Hervé Raulet 


\section{7: Saint-Malo, groupe scolaire du Grand-Bé}

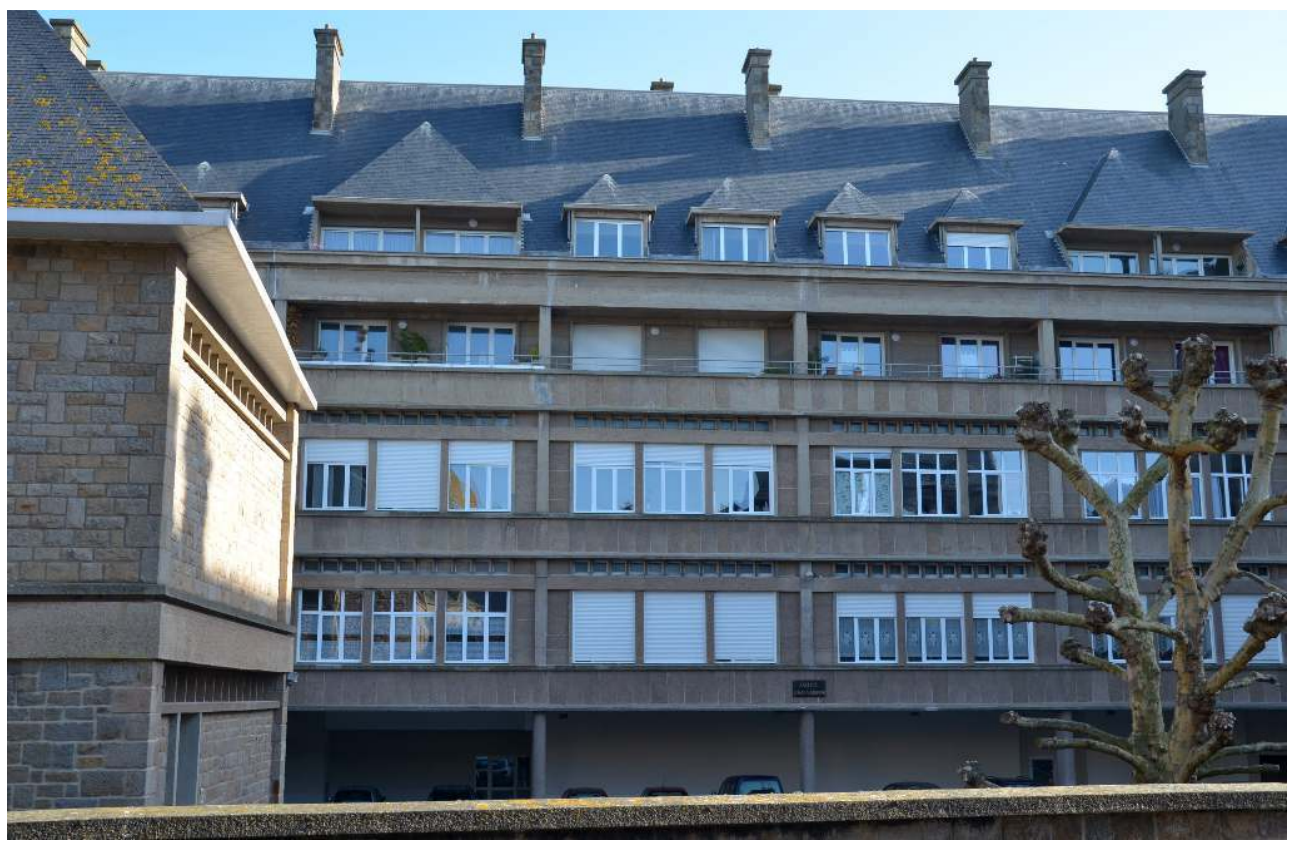

Auffret, architecte, 1951

Cl. Hervé Raulet

21 Auffret réalise, extra muros cette fois, une cité commerciale provisoire dans le quartier de Courtoisville au lendemain de la guerre ${ }^{30}$, quatre groupes scolaires, un nouvel hôpital, plusieurs équipements sportifs, ainsi qu'un nouveau casino municipal (avec Louis Arretche), actuel Palais du Grand Large, qui remplace en 1956 le bâtiment des frères Perret (1898) détruit par les bombardements (ill. 8). 


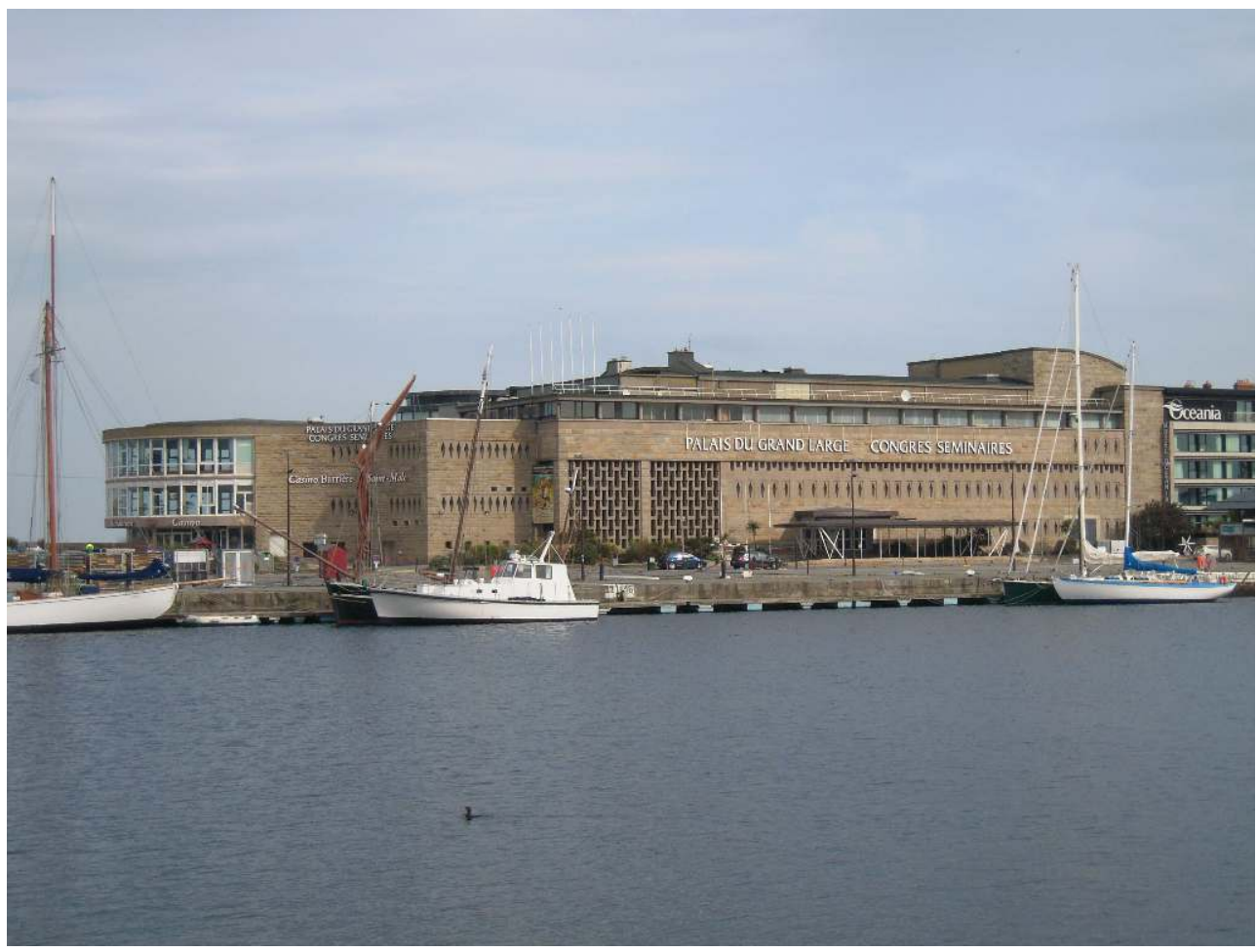

Arretche et Auffret, architectes, 1956

Cl. Hervé Raulet

22 Ces opérations témoignent d'une conception épurée et rationnelle de l'architecture qui tient compte du contexte d'insertion, comme dans le cas du casino dont la masse de granite brun prolongée d'une rotonde vitrée s'offre en contrepoint au château médiéval lui faisant face, tels deux immenses vaisseaux de pierre échoués sur le rivage. En dehors de Saint-Malo, cet architecte très prolifique et peu intéressé par la commande privée intervient dans de nombreux immeubles et programmes urbains, notamment à Brest où il élabore les plans directeurs de la ZUP de Bellevue (1958-1977), à Quimper (ZUP de Kermoysan de 1960 à 1977), Vannes (ZUP de Ménimur de 1963 à 1979, théâtre municipal, collège) ou Rennes (immeubles de logements), ainsi qu'en Normandie et en Afrique (complexes touristiques).

\section{Les phares d'Henry Auffret et de Joël Hardion}

Les phares construits par Henry Auffret, associé dans cette entreprise à Joël Hardion, sont d'une grande diversité, que ce soit en termes de catégorie de feu, de taille ou d'implantation (à terre, en mer), que de formes architecturales. Si l'on met de côté le cas particulier du phare des Héaux de Bréhat, ouvrage majeur de Léonce Reynaud dont seule la partie supérieure fut détruite et l'objet d'une reconstruction «à l'identique ", six phares sont totalement remplacés par Auffret et Hardion: le Grand-Jardin près de SaintMalo, le Paon et le Rosédo sur l'île de Bréhat, Bodic à Lézardrieux, les Sept-Iles au large de Perros-Guirec et enfin les Roches-Douvres situé à mi-route des îles de Bréhat et de Guernesey. Les phares du Paon et du Rosédo (1947-1949) remplacent deux anciennes maisons-phares construites vers 1860 . Les nouveaux ouvrages, analogues du point de vue 
de la taille et de l'inspiration régionaliste avec leur tourelle rappelant celle d'un petit manoir breton, reprennent le principe de la maison-phare, mais alors que la tour du Rosédo est associée à une véritable maison néo-bretonne en granite, celle du Paon en est privée, les architectes se servant de contreforts placés sur la face arrière du phare pour évoquer, tels les vestiges remaniés d'anciens murs, un logis disparu dont seul subsisterait la tour d'escalier. Ce rappel mémoriel de l'ouvrage détruit est également à l'œuvre au phare de Bodic (1945-1948), feu directionnel juché au sommet d'une tour en bordure de l'estuaire du Trieux ${ }^{31}$, non loin des bâtiments de la subdivision des phares de balises de Lézardrieux (ill. 9).

\section{9: Phare de Bodic}

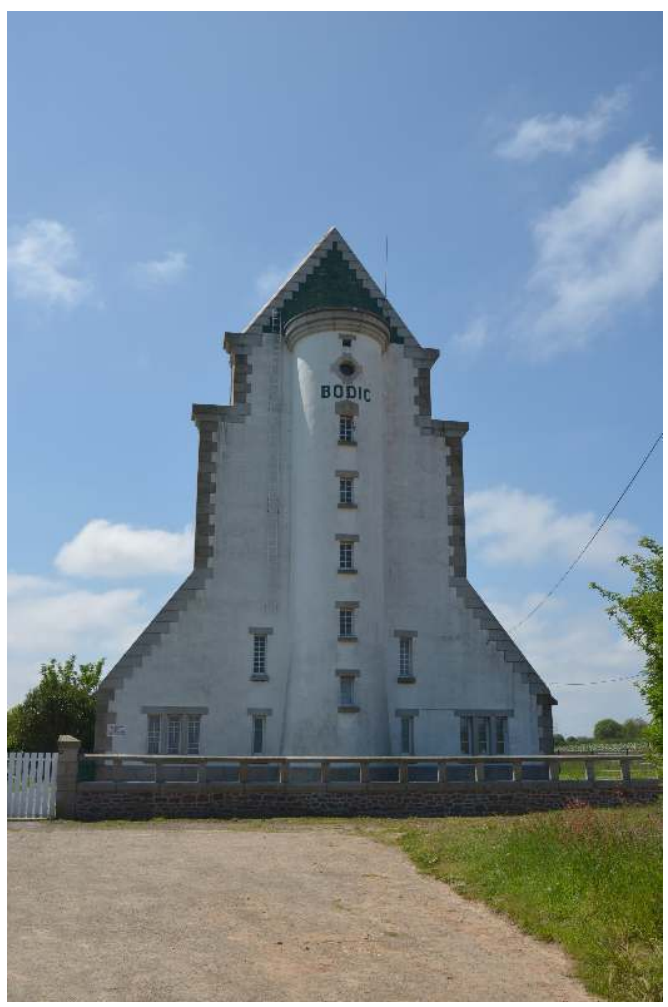

Auffret et Hardion, architectes, 1948

Cl. Hervé Raulet

La forme de l'ancien phare du XIX ${ }^{e}$ siècle, tour quadrangulaire de six niveaux couverte d'une toiture en bâtière et contenant l'ensemble des pièces techniques et de logement, est reprise en dissociant la tour de ses pièces intérieures: la première est traitée comme une très haute façade à fronton-pignon incorporant une tour d'escalier demi hors-œuvre qui permet d'accéder au feu fixe logé dans l'épaisseur de l'édifice, celui-ci voyant son ancienne fonction d'amer non seulement conservée mais augmentée par l'importante surface de mur ainsi déployée; les secondes sont, comme à Rosédo, regroupées dans une maison en granite attenante au pied de la tour, d'un style néo-renaissance bretonne. Le nouveau phare de Bodic apparaît alors comme une intéressante transposition, non seulement de la maison-phare mais également de ces églises bretonnes à clocher-mur que l'on rencontre fréquemment dans les villages de la région ${ }^{32}$ et dont certaines remplissent aujourd'hui encore une fonction d'amer. Le phare de Lézardrieux et les deux ouvrages bréhatins ont ainsi en commun de renouer avec un style «breton» que n'aurait sans 
doute pas renié Yves Hémar, avec sa part de pittoresque associé aux deux catégories d'édifices emblématiques des architectures régionales que sont la maison (et ses formes dérivées tel le manoir) et l'église.

La problématique est sensiblement différente avec le phare du Grand-Jardin en baie de Saint-Malo, même si l'on est encore une fois en présence d'une reconstruction directement inspirée de l'ouvrage détruit.

\section{10: Phare du Grand-Jardin en construction, 1948}

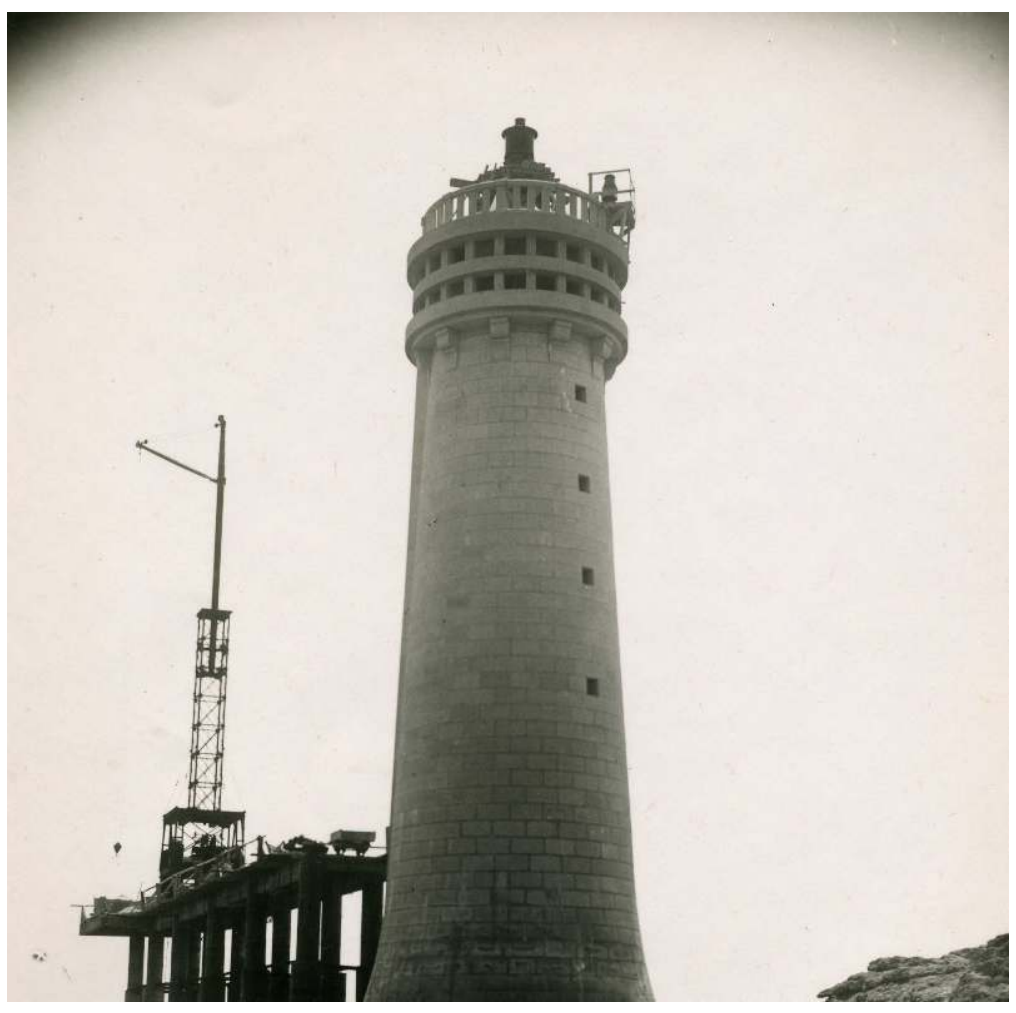

Cl. DIRM-NAMO, subdivision phares et balises Lézardrieux

Allumé en mars 1949 après deux ans de travaux (ill. 10), ce grand phare de trente mètres construit sur un rocher le plus souvent immergé pour signaler les deux chenaux d'accès au port, reprend la forme générale en " tronc d'arbre » de la tour précédente ${ }^{33}$, tout en l'élevant d'une dizaine de mètres supplémentaires. Aucune allusion régionaliste au Grand-Jardin, mais la volonté de l'inscrire avec rationalité dans les pas des ingénieurs du $\mathrm{XIX}^{\mathrm{e}}$ siècle, d'autant plus nécessaire que les contraintes d'un ouvrage de pleine mer imposent de se conformer à une typologie éprouvée de longue date. Mais Auffret et Hardion actualisent le modèle en superposant à la tour appareillée de granite un couronnement en ciment armé à l'intérieur duquel ils logent la salle de veille. Bien que l'emploi de la pierre de taille ait été un temps envisagé, l'usage du béton - y compris pour la figure mythologique de Poséidon dominant la travée principale, réalisée en ciment moulé - s'est finalement avéré judicieux, procurant à la tour cette touche moderniste conforme à l'esprit de la reconstruction (ill. 11). 
III. 11: Phare du Grand-Jardin

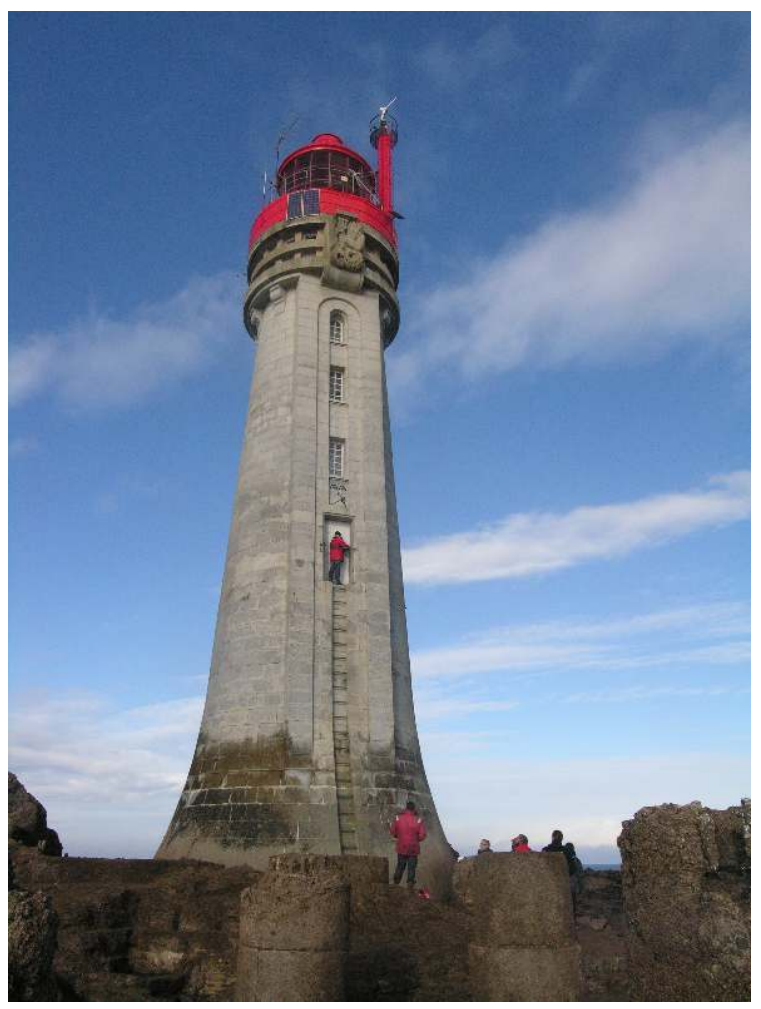

Auffret et Hardion, architectes, 1949

Cl. Hervé Raulet

L'intérieur est, en raison de l'exiguïté des volumes disponibles, dans le droit fil des phares en mer du XIX ${ }^{e}$ siècle, mais on trouve ici un aménagement marqué par l'empreinte moderniste d'un Le Corbusier et très soucieux du confort des hommes à bord, en particulier par l'emploi répété des cloisons courbes en dalles de verre qui laissent passer la lumière et procurent une sensation accrue d'espace. Même qualité de mise en œuvre dans la salle de veille dont la disposition évoque celle d'une table d'astronomie, entièrement carrelée d'une mosaïque où figure une rose des vents accompagnée des symboles du zodiaque. On assiste avec ce phare à la conception d'un ouvrage moderne qui reprend avec subtilité une formule initiale tout en l'améliorant, par une sorte de prolongement sans rupture. Il est vrai qu'un phare reste un phare et que l'on est avec ce type d'ouvrage en mer dans une logique de programme généralement très contraint, tant sur le plan technique que financier, mais une autre réalisation d'Auffret et Hardion, le phare des Roches-Douvres, montre qu'une certaine forme de démesure n'est pas exclue même dans le cas d'un ouvrage situé loin des côtes.

28 Le phare des Roches-Douvres, dont la construction débute en 1950 pour une mise en service le $1^{\text {er }}$ juin 1954, est le dernier grand établissement de signalisation maritime de Bretagne. C'est aussi le phare français le plus éloigné du continent en même temps que l'un des plus prestigieux. Situé à seize milles nautiques ${ }^{34}$ au nord-est de l'île de Bréhat, il signale un ensemble d'écueils particulièrement dangereux, générateur de déferlantes très violentes, le plateau de "Roquedouvre ». Ce phare de cinquante-huit mètres remplace un ouvrage en métal conçu par Léonce Reynaud et mis en service en 1869, dont le principe de préfabrication avait été jugé particulièrement adapté à l'éloignement et à l'inhospitalité 
du site ${ }^{35}$. Le nouveau phare adopte un mode de construction classique, fait de pierres de taille utilisées en parement (granite rose de Ploumanac'h ainsi qu'un peu de gris de Trégastel) et de béton, la provenance de ces pierres, tirées d'un immense stock amassé par les Allemands durant la guerre, autorisant une grande qualité d'exécution en même temps qu'un coût de mise en œuvre comparable à celui du projet d'origine, qui prévoyait un corps de logis en simples moellons assisés et une tour en ciment ${ }^{36}$ (ill. 12 et 13).

\section{12: Phare des Roches-Douvres}

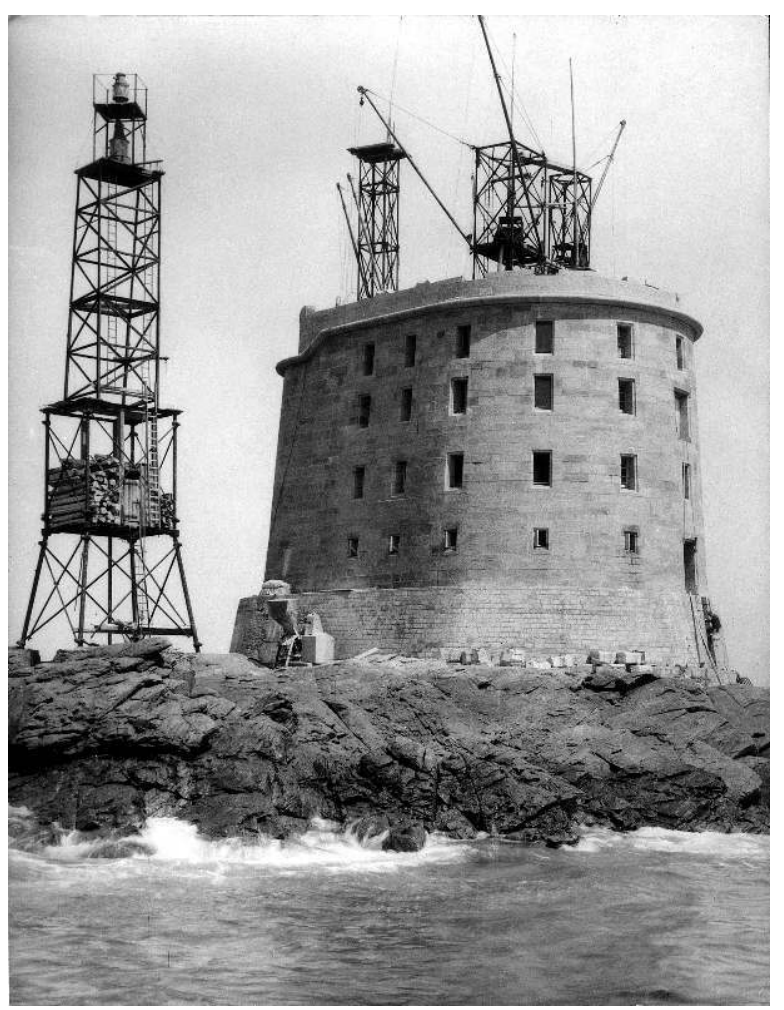

Auffret et Hardion, architectes ; en construction, 1951

Cl. DIRM-NAMO, subdivision phares et balises Lézardrieux 


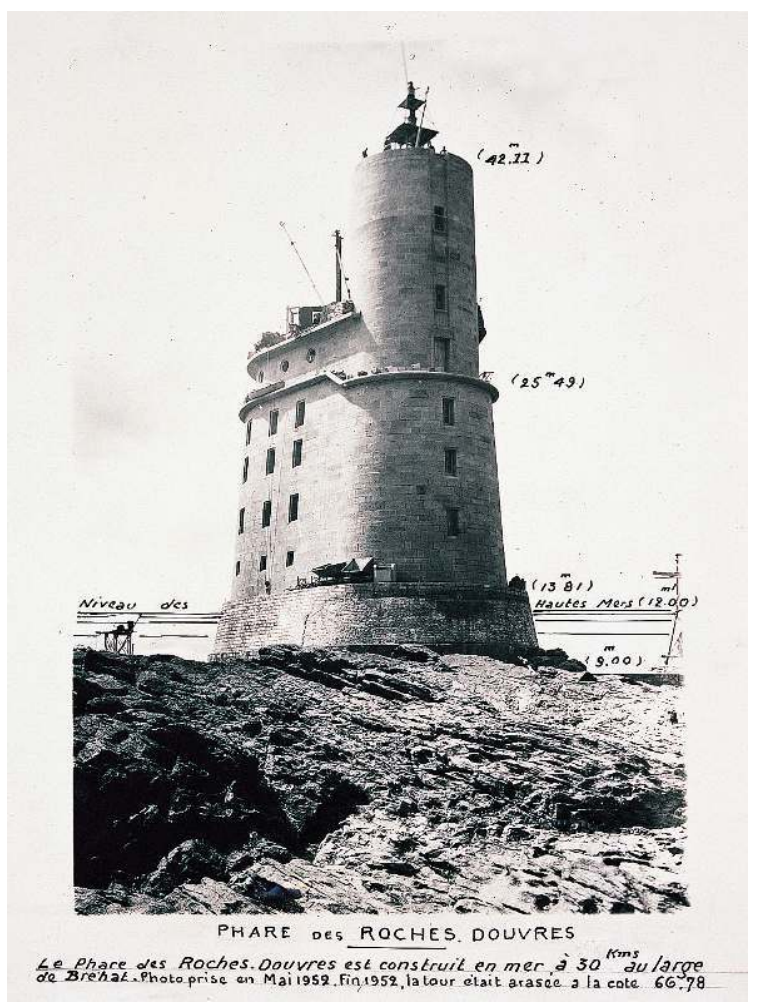

Auffret et Hardion, architectes ; en construction, mai 1952

Cl. DIRM-NAMO, subdivision phares et balises Lézardrieux

L'édifice, qui repose sur un socle en pierre et béton provenant pour partie de l'ancien phare, est d'une ampleur et d'un confort inégalés pour un ouvrage de ce type: cinq étages habitables, une cuisine et des chambres spacieuses, des salles de bain, plusieurs magasins, etc., le tout éclairé par soixante-quinze fenêtres aux menuiseries en bois exotique. Un véritable palace en comparaison de n'importe quel autre phare en mer, et en particulier du précédent Roches-Douvres, dont les pénibles conditions d'existence à bord l'avaient fait surnommer «la tour infernale ». Les raisons d'un tel programme sont sans doute à rechercher dans la volonté d'afficher, au lendemain de la guerre, le savoir-faire des ingénieurs français et d'offrir aux gardiens un environnement sain, spacieux et confortable, digne de la nouvelle ère qui s'ouvrait, mais aussi de disposer d'un avantposte stratégique d'une capacité suffisante pour accueillir, si nécessaire, d'autres hommes que les seuls agents des phares et balises...

Les premières esquisses proposées dès 1945 par les ingénieurs du Service des phares envisagent un édifice à tours accolées. L'idée est reprise par Henry Auffret qui propose finalement un ouvrage à tour excentrée dont l'avant-projet est longuement défendu par l'ingénieur en chef H. Condemine dans un rapport adressé le 5 juillet 1946 au directeur des phares André de Rouville ${ }^{37}$ :

« Nous avons acquis la conviction que l'avant-projet que nous présentons concilie à la fois l'harmonie dans le cadre, la robustesse contre les éléments et la facilité pour la construction [...].

L'allure de l'ensemble est majestueuse. La silhouette du phare, telle qu'elle est esquissée, nous paraît séduisante tant par ses lignes et ses proportions, que par l'idée même qui l'a inspirée. Le contour du soubassement a été simplifié et accentué 
au niveau des étages supérieurs évoquant dans sa partie sud la forme de la proue d'un navire.

Il convenait tout d'abord de donner à l'ouvrage projeté un soubassement massif qui constituât à la fois habitation et protection, dont la partie architectonique devait prolonger et surélever la roche naturelle, tout en conservant une transition entre la nature et la tour proprement dite, qui ne peut être conçue pratiquement et économiquement qu'en béton [...]. Tout le soubassement devra être traité en moellons, en parements d'aspect rustique, formant d'autant plus corps avec le rocher que les éléments pourront être extraits à pied d'œuvre. Sur la façade principale, au dessus de la galerie, il a été prévu un volume simple avec inscription ou motif sculpté s'encadrant dans une forme verticale formant transition esthétique entre le soubassement et le fût. Cet élément de transition nous paraît indispensable au point de vue composition [...].

Le fût de l'ouvrage devrait être traité différemment. L'aspect du béton armé de teinte plutôt claire est très admissible sinon désirable pour constituer amer. Le béton s'impose par ailleurs pour des raisons techniques. S'il ne fallait pas, pour cette partie de l'ouvrage, tomber dans la banalité des cheminées d'usine, il y avait lieu par contre d'accuser une silhouette verticale à la construction, c'est pourquoi il a été prévu des bandes verticales en béton translucide ${ }^{38}$, intégré dans le corps du béton armé, qui procureront en outre l'avantage d'éclairer convenablement l'escalier et la cage du monte-charge. Ces bandes verticales seront disposées diamétralement selon les quatre azimuts.

La pièce située à la partie haute serait largement vitrée en verre dalle. Le débordement de cette salle sur le fût, agrémenté de quatre corbeaux et accidenté de bandeaux horizontaux, permettra un couronnement caractéristique. L'ensemble devra être très foncé et traité soit en granit noir, soit en béton teinté dans la masse. »

31 L'avant-projet d'Henry Auffret, tel que présenté dans ce rapport et dans les dessins qui l'accompagnent, se différencie en plusieurs points de l'ouvrage finalement construit, non pas dans ses dispositions d'ensemble mais dans sa mise en œuvre, en particulier celle de la tour. La silhouette générale du phare est conservée, avec son grand vaisseau de granite de forme oblongue au fruit prononcé, qui évoque à la fois une forteresse maritime ${ }^{39}$ et la passerelle d'un navire avec ses hublots et son bastingage, même si certains attributs explicites comme la figure de proue (l'« élément de transition » du rapport) ont disparu. Mais l'emploi généralisé de la pierre de taille, s'il unifie l'aspect extérieur du phare, supprime du même coup l'intéressante hiérarchie des matériaux initialement prévue et qui constituait une marque essentielle de la composition. La superposition d'un massif en pierre et d'une tour en ciment ancrait le phare dans un héritage constructif traditionnel tout en revendiquant son appartenance à la modernité, ainsi que le faisait déjà, à sa manière, la tour du Grand-Jardin avec son couronnement. La tour des Roches-Douvres, finalement appareillée de granite roux et gris, reste néanmoins d'une qualité remarquable, accentuant le caractère minéral, épuré et presque militaire d'un bâtiment qui s'apparente ainsi davantage encore à l'ouvrage d'un ingénieur. CEuvre exceptionnelle à bien des égards, le phare des Roches-Douvres conclut en point d'orgue la participation d'Henry Auffret et de Joël Hardion à l'entreprise de reconstruction des phares en Bretagne.

\section{Diversité et conservatisme}

La Bretagne regroupe à elle seule plus de la moitié des phares français et l'image culturelle, touristique ou économique de cette région est, plus que tout autre sans doute, 
associée à ces ouvrages ${ }^{40}$. Au-delà de ce particularisme quantitatif ou symbolique, il n'existe pas de véritable spécificité bretonne en matière de phares, les premiers modèles architecturaux ayant été, dès la fin du XVII ${ }^{\mathrm{e}}$ siècle, indifféremment appliqués aux diverses côtes françaises, et les ouvrages construits à partir des années 1825 dans le cadre du programme national d'éclairage des côtes reflétant, par leur indifférence aux particularismes locaux, la volonté centralisatrice du Service des phares. Une région richement dotée comme la Bretagne se trouve donc logiquement porteuse d'une grande variété d'ouvrages. Même le granite, pierre éminemment bretonne et commune à l'ensemble des phares construits dans la région jusqu'au $\mathrm{XX}^{\mathrm{e}}$ siècle, se décline ici en multiples provenances, couleurs (gris, brun, "rose", noir, enduit, peint, etc.) et qualités d'appareillage, contribuant à son tour à la diversité des apparences.

Cette variété n'échappe pas, on l'a vu, aux phares reconstruits au lendemain de la seconde guerre mondiale, la collaboration des deux (trois) architectes bretons avec les ingénieurs issus du corps des Ponts et chaussées n'ayant pas conduit à une série d'ouvrages véritablement imprégnés de références régionales: le phare de Fréhel, pourtant dessiné par un architecte emblématique du néo-régionalisme de l'entre-deux-guerres, ne possède pas d'attributs spécifiquement régionaux mais exprime une forme de syncrétisme architectural dont les références puisent davantage du côté de l'Italie et des grands ouvrages du XIX siècle, et ce sont les petits feux d'Henry Auffret à Bréhat et Bodic qui affichent le plus d'accointance avec un "style breton", manière peut être pour leur architecte, jeune encore et peu suspect de nostalgie envers les préoccupations stylistiques de la première moitié du $\mathrm{XX}^{\mathrm{e}}$ siècle, de se placer malgré tout, avec distance, dans une forme de continuité historique dans cette région du Trégor particulièrement riche en édifices anciens. S'il y a un point commun à tous ces phares de la reconstruction, c'est encore une fois l'emploi exclusif ou dominant du granite comme matériau de gros œuvre ou de parement, au détriment parfois de pierres locales de bonne qualité ${ }^{41}$ et surtout du béton, qui n'arrive jamais à s'imposer. Cet emploi souvent ostentatoire du granite traduit la préférence des ingénieurs et responsables des phares et balises pour un matériau dont ils connaissent la solidité et le comportement dans le temps, d'autant que le granite est facilement disponible et sa mise en œuvre parfaitement maîtrisée. Ce choix en quelque sorte raisonnable va à l'encontre d'une certaine forme d'innovation architecturale, comme on l'a vu pour la tour des Roches-Douvres et comme ce fut également le cas pour la partie supérieure du phare des Héaux de Bréhat ${ }^{42}$. Au-delà de l'unité matérielle ainsi produite, c'est peut-être une forme de tempérance, pour ne pas dire de conservatisme prudent, qui constitue le trait commun de ces phares de la reconstruction bretonne.

\section{NOTES}

1. Bibliographie utilisée pour cet article: Isabelle Barbedor, Gaëlle Delignon, Véronique Orain, Jean-Jacques Rioult, La Côte d'Émeraude : la villégiature balnéaire autour de Dinard et Saint-Malo, Paris, Cahiers du patrimoine $\mathrm{n}^{\circ} 60,2001,340$ p.; Patrick Dieudonné dir., Bretagne $X X^{e}$, un siècle d'architectures, Saint-Brieuc, 2001, 255 p.; Francis Dreyer, Jean-Christophe Fichou, L'Histoire de tous les phares de France, Rennes, 2005, 371 p. ; Jean-Christophe Fichou, Noël Le Hénaff, Xavier 
Mével, Phares, histoire du balisage et de l'éclairage des côtes de France, Douarnenez, 2003, 452 p.; Vincent Guigueno, Au Service des phares, la signalisation maritime en France XIX ${ }^{e}-X^{e}$ siècles, Rennes, 2001, 257 p. ; Vincent Guigueno, Les phares, gardiens des côtes de France, Paris, 2012, 128 p. ; Daniel Le Couédic, Les Architectes de l'idée bretonne 1904-1945, Rennes, 1995, 911 p.; Daniel Raës, L'Architecture des phares, Saint-Malo, 1992, 440 p.; Périg Bouju, «Le Rocher de la discorde, architecture et lieux de pouvoir à Saint-Malo, $\mathrm{XIX}^{\mathrm{e}}-\mathrm{XX}^{\mathrm{e}}$ siècles ", Mémoires de la Société d'histoire et d'archéologie de Bretagne, t. LXXXIX, Rennes, 2001, p. 141-168 ; Philippe Petout, « La reconstruction de la cité historique de Saint-Malo ou le débat d'une forme en puissance contre une forme imitée ", Mémoires de la Société d'histoire et d'archéologie de Bretagne, t. LXXXIX, Rennes, 2001, p. 169-186; Roland Vidal, La construction paysagère d'une identité territoriale, imaginaire et réalité dans une station balnéaire des Côtes d'Armor, Sables-d'Or-les-Pins, thèse de doctorat en sciences de l'environnement, École nationale du génie rural et des eaux et forêts, 2003, 281 p.; Catherine Lebret, L'œuvre d'un architecte néo-régionaliste: Yves Hémar, mémoire de maîtrise, sous la dir. de François Loyer, université Rennes 2, 1985, 80 p.; Anne Lecerf-Daufin, Yves Hémar (1886-1955), architecture privée à Saint-Malo, mémoire de maîtrise, sous la dir. de Jean-Yves Andrieux, université Rennes 2, 2002, $100 \mathrm{p}$.

2. Son appartenance à des associations culturelles affiliées au mouvement régionaliste breton Emsav lui valut une mise à l'écart après la guerre.

3. îlots $n^{\circ} 1$ (rue Guy Louvel), $n^{\circ} 55$ (rue Saint-Vincent) et $n^{\circ} 58$ (rue Jacques Cartier).

4. Devient en mai 1947 architecte en chef de la reconstruction de Caen.

5. Bâtiment démoli en 1985.

6. Il reprit du service du 20 juin 1945 à l'allumage du phare actuel le $1^{\text {er }}$ juillet 1950.

7. Ce phare en service depuis 1950 (ESM n 547/000) est le quatrième sur le cap; le troisième, construit d'après les plans des ingénieurs Méquin et Boucher remaniés par Léonce Reynaud, fonctionna de 1847 à son dynamitage le 10 août 1944.

8. Le Réveil breton, 27 juillet 1924.

9. La connaissance de l'œuvre d'Hémar est tributaire de la rareté de la documentation historique le concernant: les archives de l'architecte conservées dans son agence rue Guy Louvel ont été entièrement détruites sous les bombardements en août 1944, de même qu'une bonne partie des archives municipales de Saint-Malo, sa production concentrée sur la côte d'Émeraude n'a pas laissé de traces auprès des organismes centralisés d'architecture, et le permis de construire n'étant pas obligatoire avant-guerre, la plupart de ses constructions ne sont pas enregistrées.

10. Un second architecte, Pol Abraham (1891-1966), dont l'œuvre se révèlera beaucoup plus déterminante que celle d'Hémar, est engagé par Brouard pour ses conceptions résolument modernistes, mais il ne rencontrera pas beaucoup de succès auprès des clients de Sables-d'Or malgré la grande qualité de ses maisons.

11. Parmi les meilleurs initiateurs de ce style naissant, on peut citer Gaston Chabal déjà cité et Pierre-Jack Laloy (1885-1962).

12. Le palais de justice de Casablanca (architecte Joseph Marrast, 1913), l'hôtel des postes de Rabat (architecte Adrien Laforgue, ca 1915).

13. Lucien Vaugeois, architecte. La délégation bretonne est emmenée par le peintre Jean-Julien Lemordant.

14. Il dessine notamment le mobilier de la salle à manger du pavillon breton. Membre de la Société des arts décoratifs bretons, Hémar était un collectionneur et fin connaisseur de l'artisanat breton traditionnel (meubles, objets, costumes, etc.) : il avait créé un petit «musée d'Art breton » dans la maison natale de Duguay-Trouin dont il était propriétaire, et organise en 1927 une exposition de sa collection de plus de 3000 pièces au château de Saint-Malo.

15. La période comprise entre 1450 et le début du XVII ${ }^{\mathrm{e}}$ siècle correspond à un "âge d'or " économique de la Bretagne ayant favorisé l'émergence d'une architecture nobiliaire dont l'archétype est le manoir rural de style gothique puis Renaissance, construit en granit, avec tour 
d'escalier en vis demi hors-œuvre. Il sera supplanté au cours du XVII ${ }^{e}$ siècle par le modèle « français » de château entre cour et jardin, incorporant un escalier droit intérieur.

16. Les Lutins (St-Malo) en 1914, Château-Richeux (St-Méloir-des-Ondes) vers 1920, L'Escale (StMalo) en 1923.

17. La Vie technique et industrielle, septembre 1926.

18. L'Ouest-Éclair, 4 juin 1928.

19. Dite aussi « Les Pins » à Sables-d'Or-les-Pins.

20. La Construction moderne, février-mai 1931.

21. Le Journal, juillet 1927.

22. La seconde moitié $d u X_{X}{ }^{e}$ et le début $d u X X^{\mathrm{e}}$ siècles seront également marqués par une profusion d'édifices néogothiques dont beaucoup réinterprètent les modèles locaux de la fin du Moyen-Âge.

23. Les œuvres de Bouillé s'inscrivent dans l'influence de l'architecte et moine bénédictin dom Bellot (1876-1944).

24. Isidore Odorico (1893-1945), issu d'une dynastie de mosaïstes italiens originaire du Frioul et installée à Rennes en 1882, intervient dans de nombreux chantiers en Ille-et-Vilaine et Côtes d'Armor, ainsi qu'en Mayenne et en Maine-et-Loire.

25. La villa Bric-à-Brac (1860), l'une des premières maisons de villégiature de Dinard, construite pour Lyona Faber, résidente anglaise ayant beaucoup contribué au lancement de la station.

26. Arch. nat., $\mathrm{F}^{14} 19988$.

27. Ibid.

28. On peut citer les phares d'Ailly et de la Hève (Seine-Maritime) au XVIII ${ }^{\mathrm{e}}$ siècle, et au siècle suivant ceux de la Balue (Ille-et-Vilaine), des Triagoz (Côtes d'Armor), de la Vieille (Finistère), du Pilier (Vendée), de St-Georges de Didonne (Charente-Maritime), de la pointe de Grave et de Hourtin (Gironde), du cap Béar (Pyrénées-Atlantiques), etc.

29. Ce bâtiment conçu dès 1946 devait être le nouvel hôtel de ville, qui sera en définitive installé dans une partie de l'ancien château réaménagée par l'architecte en chef des monuments historiques Raymond Cornon assisté d'Auffret lui-même.

30. Considérée comme très novatrice en ce qu'elle préfigurait les galeries marchandes des années 1970.

31. Bodic est aligné sur le phare de la Croix situé à l'embouchure du Trieux.

32. Ces églises trégorroises ont généralement été construites au XVII ${ }^{\mathrm{e}}$ siècle; parmi les plus proches, on peut citer Lézardrieux, Pleudaniel ou encore Trédarzec.

33. Les phares britanniques d'Eddystone IV (John Smeaton, 1757-1759, détruit en 1877) et de Bell Rock (Robert Stevenson, 1807-1811) furent les premiers à expérimenter cette forme obtenue par la rotation d'une ellipse autour d'un axe vertical, particulièrement adaptée aux assauts de la houle et des déferlantes. Ce modèle est utilisé pour la première fois en France par les ingénieurs Plantier et Rapatel au phare du Four (1819-1822) situé au large du Croisic, puis par Léonce Reynaud au phare des Héaux de Bréhat (1835-1840), et ensuite appliqué à la plupart des phares en mer.

34. $30 \mathrm{~km}$ environ, ce qui le place en fait à près de $40 \mathrm{~km}$ du continent.

35. Ce premier phare reprenait le modèle du phare Amédée conçu par Reynaud et mis en service quelques années plus tôt sur un atoll au large de Nouméa (ce phare néo-calédonien est aujourd'hui le seul ouvrage métallique encore opérationnel en France).

36. Un projet alternatif fut également proposé par les Entreprises métropolitaines et coloniales, consistant en une tour cylindrique en béton non armé arrimée à un soubassement également en béton au moyen de vingt-quatre câbles Coyne disposés à l'intérieur du fût et scellés au rocher à une profondeur d'une dizaine de mètres (architecte Henri Mathé). L'incertitude quant au comportement dans le temps d'un tel ouvrage, soumis à l'action de la mer et très éloigné des 
côtes, fit renoncer l'administration, malgré un coût de mise en œuvre plus réduit que le projet classique finalement retenu.

37. Arch. nat., $\mathrm{F}^{14} 19995$.

38. On ne sait à quel matériau ou mise en œuvre l'ingénieur fait ici référence (maille de béton ajouré comme au casino municipal de Saint-Malo ?). De véritables bétons translucides existent désormais, qui reposent notamment sur l'incorporation de fibres optiques au sein du matériau.

39. On pense évidemment au fort de la Conchée édifié entre 1690 et 1705 par Jean-Siméon Garangeau en rade de Saint-Malo, au château du Taureau reconstruit par le même ingénieur en baie de Morlaix, ou encore au fort Boyard situé au large de l'île d'oléron.

40. Un exemple actuel parmi les plus explicites est le logo de la marque Produit en Bretagne créée en 1995 et regroupant deux cent soixante entreprises bretonnes productrices de biens et de services, qui représente un phare éclairant une carte de la Bretagne «historique " (actuelle région Bretagne et Loire-Atlantique).

41. Ainsi au cap Fréhel, massif pourtant constitué d'un grès rouge très résistant dont les carrières ont fourni des pierres de construction pour toute la région, à l'instar de l'ancienne tour à feu allumée en 1702.

42. Plusieurs projets alternatifs furent étudiés, dont celui de Georges Tourry qui proposa un couronnement annulaire en béton armé très débordant, qui devait contenir la chambre de veille et une salle des machines fournissant l'électricité nécessaire au fonctionnement du phare.

\section{RÉSUMÉS}

Le remplacement des phares détruits à la fin de la seconde guerre mondiale en Bretagne-Nord s'appuie sur la contribution de deux figures importantes de l'architecture régionale, Yves Hémar (1886-1955) et Henry Auffret (1919-1997), qui prennent tous deux une part active à la reconstruction de Saint-Malo. La douzaine de phares reconstruits offre une large diversité de styles où s'expriment parfois quelques références à l'architecture régionale, mais sans que l'on puisse pourtant y trouver l'affirmation d'une véritable spécificité bretonne. Seul l'emploi systématique du granite, matériau de prédilection des ingénieurs et pierre emblématique de la Bretagne, apparaît comme un trait commun à tous ces ouvrages, sa mise en œuvre souvent ostentatoire traduisant, au-delà de la recherche de durabilité et de résistance, une forme d'attachement aux principes esthétiques du siècle précédent.

The reconstruction of lighthouses on the north coast of Brittany destroyed at the end of the Second World War was largely the work of two architects, Yves Hémar (1886-1955) and Henry Auffret (1919-97). Both took an active part in the reconstruction of Saint-Malo after the war and made important contributions to regional architecture. The dozen lighthouses they rebuilt were executed in a wide variety of styles and occasionally included references to regional architecture; they did not, however, seek to emphasize a distinctive, Breton character in these buildings. Only the systematic use of granite, the material of choice for engineers and a stone emblematic of Brittany, appears to be a common feature in all these works. Beyond merely seeking strength and durability, the use of granite in these lighthouses signals an attachment to the aesthetic principles of the previous century. 
Der Ersatz der am Ende des Zweiten Weltkriegs in der Nord-Bretagne zerstörten Leuchttürme führt den besonderen Beitrag von zwei bedeutsamen Vertretern der regionalen Architektur vor Augen, namentlich Yves Hémar (1886-1955) und Henry Auffret (1919-1997), die beide an dem Wiederaufbau von Saint-Malo aktiv beteiligt waren. An dem Dutzend von Leuchttürmen, die wiederaufgebaut wurden, drücken sich verschiedene Stilformen aus, die manchmal die regionale Tradition übernehmen, aber ohne dass sich dadurch eine echte bretonische Identität erkennen lässt. Gemeinsam haben alle diese Bauten nur die systematische Anwendung des Granits, Lieblingsmaterial der Ingenieure, das auch als besonders repräsentativ für die Bretagne gilt. Über die dauerhaften und haltbaren Eigenschaften des Granits hinaus zeugt dessen oft auffällige Verwendung von der Anhänglichkeit an die ästhetische Tradition des vorigen Jahrhunderts.

\section{AUTEUR}

\section{HERVÉ RAULET}

Hervé Raulet, né en 1959, est chargé des protections d'immeubles à la Direction régionale des affaires culturelles de Bretagne (conservation régionale des monuments historiques). 